\title{
The Global Oscillation Network Group (GONG) Project
}

\author{
J. W. Harvey, F. Hill, R. P. Hubbard, J. R. Kennedy, \\ J. W. Leibacher, J. A. Pintar, P. A. Gilman, R. W. Noyes, \\ A. M. Title, J. Toomre, R. K. Ulrich, A. Bhatnagar,
} J. A. Kennewell, W. Marquette, J. Patrón, O. Saá, E. Yasukawa

Helioseismology requires nearly continuous observations of the oscillations of the solar surface for long periods of time in order to obtain precise measurements of the sun's normal modes of oscillation. The GONG project acquires velocity images from a network of six identical instruments distributed around the world. The GONG network began full operation in October 1995. It has achieved a duty cycle of 89 percent and reduced the magnitude of spectral artifacts by a factor of 280 in power, compared with single-site observations. The instrumental noise is less than the observed solar background.

The GONG project instrumentation was designed to measure the conditions in the solar interior using information from the acoustic waves that propagate through the sun. High-precision measurements of the temporal frequencies of the normal modes excited in the sun are required to infer the conditions, requiring continuous observations lasting over many mode lifetimes (typically weeks to months and longer). To obtain these data, the GONG project has deployed a network of six instruments around the world to record the Doppler velocity of the solar surface. The specification and construction of six identical instruments capable of acquiring the data with both low noise and long-term stability stringently constrained the optical, mechanical, and thermal specifications. The GONG project is now fully operational.

J. W. Harvey, F. Hill, R. P. Hubbard, J. R. Kennedy, J.W. Leibacher, and J. A. Pintar are with the National Solar Observatory, National Optical Astronomy Observatories, Post Office Box 26732, Tucson, AZ 85726-6732, USA P. A. Gilman is with the High Altitude Observatory, National Center for Atmospheric Research, Post Office Box 3000, Boulder, CO 80307, USA. R. W. Noyes is with the Smithsonian Astrophysical Observatory, 60 Garden Street, Cambridge, MA 02138, USA. A. M. Title is with the Lockheed Solar and Astrophysics Laboratory. Palo Alto, CA 94304, USA. J. Toomre is with JILA, University of Colorado, Boulder, CO 80309 , USA. R. K. Ulrich is with the Department of Physics and Astronomy, University of California, Los Angeles, CA 90024, USA. A. Bhatnagar is with the Udaipur Solar Observatory, Physical Research Laboratory, Udaipur, India. J. A. Kennewell is with the Learmonth Solar Observatory, IPS Radio and Space Services, Exmouth, Western Australia, Australia. W. Marquette is with the Big Bear Solar Observatory, Big Bear City, CA 92314, and California Institute of Technology, 26433, Pasadena, CA 91125, USA. J. Patrón is with the Observatorio del Teide, Instituto Astrofísica de Canarias, La Laguna, Tenerife, Spain. O. Saá is with the Cerro Tololo Interamerican Observatory, National Optical Astronomy Observatories, La Serena, Chile. E. Yasukawa is with the Mauna Loa Solar Observatory, Hilo, HI 96720, and High Altitude Observatory, National Center for Atmospheric Research, Post Office Box 3000, Boulder, CO 80307, USA.

\section{Scientific Objectives and Technical Requirements}

Several processes contribute to the velocity field of the solar surface: differential rotation, convective flows, circulation, and the oscillations. The oscillatory contribution consists of the incoherent superposition of roughly $10^{7}$ pressure $(p)$ modes with temporal cyclic frequencies $v$ of about 2 to $4 \mathrm{mHz}$ (that is, with periods of about $5 \mathrm{~min}$ ) and maximum velocity amplitudes of about 20 $\mathrm{cm} \mathrm{s}^{-1}$ per mode. The fundamental modes, with properties similar to surface waves over the deep terrestrial ocean, are also observed. Gravity modes should also exist but are trapped deep within the sun beneath the convection zone, and so far, observations of them at the surface have been equivocal.

The $p$ modes are most likely (i) stochastically excited by turbulence in the layers immediately below the photosphere and (ii) damped by radiative losses. The interplay of driving and damping produces an average lifetime of the modes that depends primarily on spherical harmonic degree $\ell$ and radial order $n$. The lifetimes range from a few hours for high- $\ell$, low- $n$ modes $(\ell \sim 250, n \sim 1)$ to longer than several months for modes with low $\ell$ and low $n(\ell \sim 0, n \sim 10)$.

To observe the $p$ modes, one acquires a time series of images of the Doppler velocity of the solar surface (Fig. 1A) and decomposes the images into time series of amplitudes of spherical harmonics (Fig. 1B). A temporal Fourier transform is performed, and compact displays of the observed oscillation properties are provided by various multidimensional power spectra assembled from the transform moduli (Fig. 1C). The spatial-temporal spectrum is dominated by ridges that are a consequence of the refraction of the acoustic waves by the temperature gradient in the solar interior (1). The subsequent interference of the waves trapped within the temperature cavity results in the survival of only those waves that satisfy the boundary conditions at the two depths where the wave is reflected. Each ridge corresponds to a different value of $n$; modes with higher $n$ also have higher $v$ at a constant $\ell$. At values of $\ell$ less than about 180 , the ridges are resolved into discrete features. These modes are global in nature and have lifetimes longer than their travel time around the sun (typically about 5 days). The global modes are thus able to interfere constructively, producing discrete peaks in the spectrum. The width of the peak is inversely proportional to the lifetime of the mode, and the frequency of the mode is estimated from the location of the peak in the spectrum.

The observable parameters of a mode, in particular $v$, depend on the physical conditions (primarily density, pressure, velocity, and magnetic field; and indirectly, temperature and composition) of the solar interior along the ray path of the mode. Each mode samples different regions in depth and heliographic latitude. Modes with lower values of $\ell$ and higher values of $n$ sample deeper depths than those with relatively high $\ell$ and low $n$. Thus, the physical conditions in a given volume can be estimated by comparing the parameters of modes whose ray paths differ only by the location of the volume considered. The physical conditions within the entire solar interior can then be inferred by analyzing the frequencies of a large number of modes.

Observations over long periods of time are important for two reasons. (i) The inference of the deep solar structure requires measurements of $v$ for the low-frequency modes that penetrate to the energy-generating core, and these measurements must be accurate enough to reduce random and systematic errors in the estimated physical parameters to acceptable levels. Because measurement of $v$ for a mode requires its line profile be resolved, it is necessary to observe the mode for many lifetimes. Because unresolved peaks are present in the longest data sets yet achieved, the upper limit of the lifetimes is still unknown, but some modes must have lifetimes approaching 1 year. Thus, we must observe these modes for at least a few years to determine their parameters. (ii) The frequencies of the $p$ modes vary with the solar magnetic activity cycle of about 11 years. The magnitude of the frequency shift depends on $\nu$. This systematic variation provides information on the structure of the near-surface layers as the activity cycle evolves but must be removed for determination of the deep interior structure. Accurate measurements of this shift also require long-term observations. 


\section{Observing Strategies}

Long-term observations from a single ground-based site are regularly interrupted by the daily setting of the sun, and these periodic gaps create a set of sharp side lobes surrounding each solar peak in the spectrum (Fig. 2A). The presence of these diurnal side lobes confuses the observed spectrum and has led to three observing strategies to eliminate them: observations from the South Pole (2), observations from a continuously sunlit location in space (3), and observations from a network of sites distributed around the Earth. Polar observations are the least expensive of the strategies, but they present cold-weather technical challenges, cannot last longer than about 3 months, and are vulnerable to interruptions by local weather conditions. Spaceborne observations are free of terrestrial atmospheric effects but are expensive to implement and difficult to maintain over long periods. Networks are intermediate in expense, can be repaired, and are robust to weather and instrumental interruptions, but the data contain terrestrial atmospheric effects. Multisite network data must be merged together, which complicates the data reduction but also provides verification and cross-calibration of the instruments and improves the signal-to-noise ratio of the observations.

Two integrated-light networks have been deployed: BiSON (Birmingham Solar Oscillation Network, administered by the University of Birmingham, UK) (4) and IRIS (International Research on the Interior of the Sun, administered by the Univer- sité de Nice, France) (5). These networks use atomic resonance spectrometers that view the sun as a star and are sensitive only to modes with $0 \leq \ell \leq 4$. The Taiwanese Oscillation Network (TON, administered by Tsing Hua University, Hsinchu, Taiwan) is being deployed (6). This network

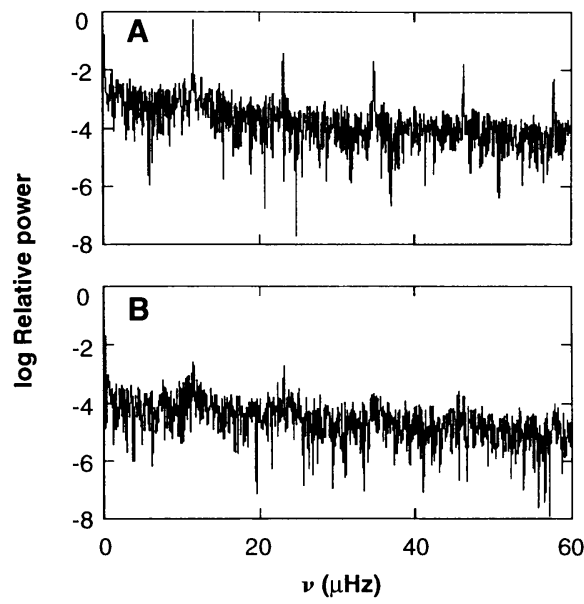

Fig. 2. (A) Power spectrum of the temporal observing window obtained over 4 months at a single site, normalized to the power at zero frequency. The set of sharp features at integral multiples of $11.57 \mu \mathrm{Hz}$ (1 per day) are caused by the daily setting of the sun, which produces regularly spaced gaps in the data. This spectrum is replicated around each solar oscillation peak, greatly increasing the number of features in the power spectrum and complicating the identification and determination of the frequencies of the $p$ modes (B) Power spectrum of the temporal observing window of the GONG network for a similar length of time. The diurnal side lobes have been reduced in power by a factor of 280 . will acquire 1242-pixel by 1152-pixel images of solar brightness using a narrowband filter. Finally, a small network of high-degree Doppler velocity imaging instruments is being deployed in California and the former Soviet Union (7).

The selection of the sites for GONG began with computer modeling showing that a properly placed six-site network could achieve an observational duty cycle of over $90 \%$, reducing the amplitude of the diurnal side lobes by more than two orders of magnitude (8). This was confirmed by a survey of the actual conditions for solar observing at 15 candidate sites by small sunshine monitors (9). Six of these sites were finally chosen for the GONG network (Fig. 3). The site survey data demonstrated that a duty cycle of $93 \%$ was achievable at

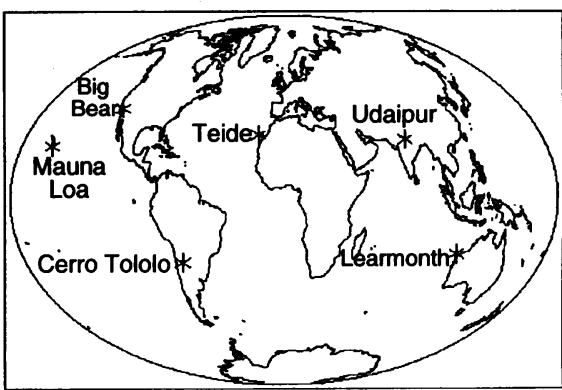

Fig. 3. Location of the observing sites comprising the GONG network. There are six sites: Big Bear Solar Obsenvatory, California, USA; Mauna Loa Solar Observatory, Hawaii, USA; Learmonth Solar Observatory, Australia; Udaipur Solar Observatory, India; Observatorio del Teide, Tenerife, Spain and Cerro Tololo Interamerican Observatory, Chile.
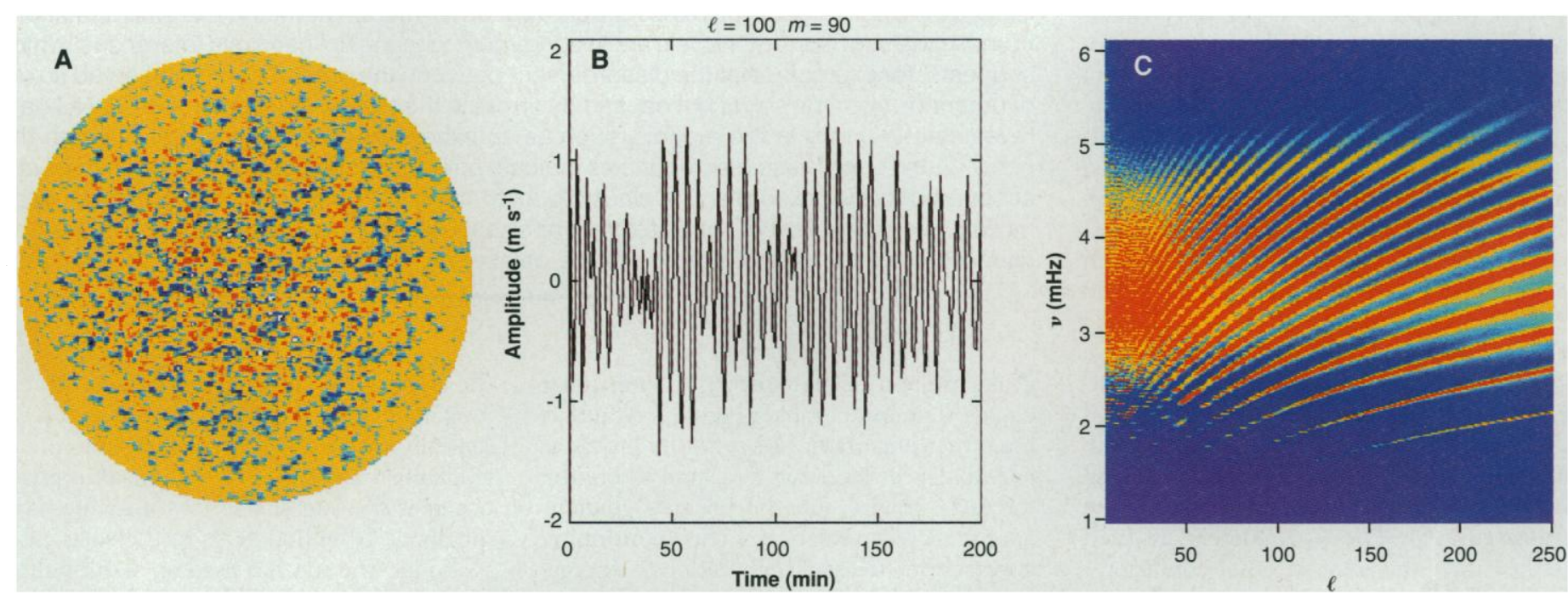

Fig. 1. An overview of information flow in helioseismology. (A) Doppler velocity image of the solar surface as observed by a GONG instrument. A high-pass temporal filter has been applied to reveal a snapshot of the oscillatory velocity field. (B) The decomposition of many velocity images into spherical harmonics produces a time series of the superposition of spherical harmonic amplitudes for modes with the same values of degree $\ell$ and azimuthal order $m$ and several different values of radial order $n$, each with a different temporal frequency $\boldsymbol{v}$.

Shown here is a portion of the resulting time series for $\ell=100, m=90$. (C) A temporal Fourier transform of the time series separates the modes with different $v$. The resulting power spectra can be averaged over $m$ to produce this two-dimensional power spectrum of the Doppler velocity of the solar surface as a function of $\ell$ and $v$. The ridges in the diagram correspond to different values of $n$, and the location of the ridges in the spectrum are used to infer the internal solar conditions. 
these sites, and the first month of full operations yielded a duty cycle of $89 \%$. The first diurnal side lobe has been reduced by a factor of 280 in power (Fig. 2B).

\section{The GONG Instrument and Data Reduction}

The GONG instrument (10) was designed to observe the Doppler shift of the solar absorption line of $\mathrm{Ni}$ i (at wavelength 676.8 $\mathrm{nm}$ ) primarily for $p$ modes. Doppler velocity measurements were chosen because they typically have a higher ratio of the oscillatory signal compared to other solar processes. The actual measurement is of the phase of the single strongest (on average) Fourier component in a small portion of the spectrum around one absorption line. Such instruments are known in the solar community as "Fourier tachometers" (11). In the optics community, the basic technique is known as phase-shift interferometry and is widely used to test the quality of optical components. All of the instrumental design goals have been met or exceeded; in particular, the noise level of the measurements is two orders of magnitude below the noise generated by nonoscillatory solar processes.

Each minute, a single site records three 256-pixel by 242-pixel images, a total of 393 kilobytes of information. On average, 1.8 sites observe concurrently, so the average total data rate for the network is over 1 gigabyte per day. In order to maintain cadence, data must be reduced and archived at the same overall rate as they are collected. Many of the processing steps are independent, so the computational burden has been distributed over a network of workstations. This approach allows parallel processing of several steps and allows data reduction to maintain cadence with the data collection (12).

The images are processed, filtered, calibrated, and converted to Doppler velocity, modulation (approximate line strength), and total intensity images (13). The spherical harmonic decomposition of each remapped image is estimated up to $\ell=250$ (14), thereby sampling all of the globally resonant modes. Time series of the mode amplitudes obtained simultaneously at different sites are corrected and combined (15), and the power spectra are computed. Finally, the frequencies, amplitudes, and linewidths of peaks in the power spectra are estimated (16). These parameters are then used to infer the solar internal conditions. The GONG network will provide this information for at least the next 3 years.

\section{REFERENCES AND NOTES}

1. The original papers providing the theoretical underpinnings of the oscillations are R. Ulrich, Astrophys. J. 162, 993 (1970); J. W. Leibacher and R. F. Stein
Astrophys. Lett. 7, 191 (1971); C. L. Wolff, Astro phys. J. 177, L87 (1972). For more recent reviews of the subject, see D. O. Gough and J. Toomre, Annu. Rev. Astron. Astrophys. 29, 627 (1991); A. N. Cox, W. C. Livingston, M. S. Matthews, Eds., Solar Interior and Atmosphere (Univ. of Arizona Press, Tucson, 1991), pp. 329-660; W. Unno, Y. Osaki, H. Ando, H. Saio, H. Shibahashi, Nonradial Oscillations of Stars (Univ. of Tokyo Press, Tokyo, 1989).

2. J. W. Harvey, AIP Conf. Proc. 189, 227 (1989).

3. P. H. Scherrer et al., Sol. Phys. 162, 129 (1995)

4. Y. Elsworth et al., Astron. Soc. Pac. Conf. Ser. 76, 392 (1995).

5. E. Fossat, Sol. Phys. 133, 1 (1991).

6. D. Y. Chou et al., ibid. 160, 237 (1995).

7. E. J. Rhodes Jr. et al. , Astron. Soc. Pac. Conf. Ser. 76, 398 (1995)

8. F. Hill and G. Newkirk Jr., Sol. Phys. 95, 201 (1985).

9. F. Hill et al., ibid. 152, 321 (1994); ibid., p. 351.

10. J. W. Harvey et al., in preparation.

11. J. Beckers and T. M. Brown, Oss. Mem. Oss. Astrofis. Arcetri 106, 189, (1978)

12. F. Hill et al., in preparation.

13. R. Toussaint, J. W. Harvey, R. P. Hubbard, Astron. Soc. Pac. Conf. Ser. 76, 532 (1995)

14. T. Brown, in Advances in Helio- and Asteroseismol- ogy, J. Christensen-Dalsgaard and S. Frandsen, Eds. (Reidel, Dordrecht, 1988), pp. 453-465

15. The time series from each site-day, scaled to compensate for variations in image resolution from seeing and other effects, are merged to form a longer, more nearly continuous time series. The uncertainty in the scaling estimate is used as a measure of data quality to weight the time series W. Williams, F. Hill, C. Toner, T. Brown, Astron. Soc. Pac. Conf. Ser. 42, 441 (1993); W. Williams, F. Hill, C. Toner, ibid. 52, 494 (1993); W. Williams, C. Toner, F. Hill, ibid. 76, 500 (1995); in Fourth SOHO Workshop: Helioseismology Volume 2, J. T. Hoeksema, V. Domingo, B. Fleck, B. Battrick, Eds. (European Space AgencyPubl. Div., Noordwijk aan Zee, Netherlands, 1995), pp. 185-189]. Merging algorithms have also been developed in the context of nonimaged helioseismology for the IRIS network [E. Fossat, Astron. Astrophys. 263, 443 (1992)].

16. F. Hill et al., Science 272, 1292 (1996).

17. The GONG project is managed by the National Solar Observatory, a Division of the National Optical Astronomy Observatories, which is operated by the Association of Universities for Research in Astronomy under a cooperative agreement with NSF.

18 March 1996; accepted 6 May 1996

\section{The Current State of Solar Modeling}

J. Christensen-Dalsgaard, W. Däppen, S. V. Ajukov, E. R. Anderson, H. M. Antia, S. Basu, V. A. Baturin, G. Berthomieu, B. Chaboyer, S. M. Chitre, A. N. Cox, P. Demarque, J. Donatowicz, W. A. Dziembowski, M. Gabriel, D. O. Gough, D. B. Guenther, J. A. Guzik, J. W. Harvey, F. Hill, G. Houdek, C. A. Iglesias, A. G. Kosovichev, J. W. Leibacher, P. Morel, C. R. Proffitt, J. Provost, J. Reiter, E. J. Rhodes Jr., F. J. Rogers, I. W. Roxburgh, M. J. Thompson, R. K. Ulrich

Data from the Global Oscillation Network Group (GONG) project and other helioseismic experiments provide a test for models of stellar interiors and for the thermodynamic and radiative properties, on which the models depend, of matter under the extreme conditions found in the sun. Current models are in agreement with the helioseismic inferences, which suggests, for example, that the disagreement between the predicted and observed fluxes of neutrinos from the sun is not caused by errors in the models. However, the GONG data reveal subtle errors in the models, such as an excess in sound speed just beneath the convection zone. These discrepancies indicate effects that have so far not been correctly accounted for; for example, it is plausible that the sound-speed differences reflect weak mixing in stellar interiors, of potential importance to the overall evolution of stars and ultimately to estimates of the age of the galaxy based on stellar evolution calculations.

Stars are born from contracting interstellar clouds. The initial rapid phases of evolution are rather uncertain; however, the protostar eventually settles down to a state where the forces of gravity and the pressure gradient approximately balance. The continuing contraction releases gravitational energy, which heats up the stellar matter and supplies the luminosity of the early star. Eventually, the temperature in the stellar core gets sufficiently high that the energy released in the fusion of hydrogen into helium begins to contribute to the luminosity. Contraction stops when fusion produces all of the luminosity. The star then enters the very long main-sequence phase, during which essentially all of the hydrogen in the core is gradually converted into helium. This phase occupies the largest fraction of its life, lasting about 10 billion years in the solar case.

So far, the sun has used up about half of its hydrogen supply. When the hydrogen at the center is exhausted, in about 5 billion years, fusion will continue in a shell around the helium core. During this phase, the sun will expand greatly, becoming finally a red giant. The sun will end as a white dwarf, comprising much of the original mass but 